Moroccan J. of Pure and Appl. Anal. (MJPAA)

Volume 4(2), 2018, Pages 85-93

ISSN: Online 2351-8227 - Print 2605-6364

DOI 10.1515/mjpaa-2018-0009

\title{
The Upper and Lower Approximations in Rough Subgroupoid of a Groupoid
}

\author{
H. TASBOZAN ${ }^{1, a}$ AND I. ICEN ${ }^{2, b}$
}

Авstract. In this article, we introduce the concept of rough subgroupoid of a groupoid as a generalization of a rough subgroup and give some features about the lower and the upper approximations in a groupoid [1]. We give some of the characterization of them. Key words and phrases. rough set, rough groupoid, rough subgroupoid.

\section{Introduction}

The rough set is not only concerned with uncertainty. It is also effective in the calculation methods of soft clusters. These areas include artificial intelligence, data mining, pattern recognition, decision analysis and fault diagnosis. The concept of a rough set was introduced by Pawlak in [2]. The algebraic approach of rough set was studied by some authors $[1,3,4,5,6,7,8,9,10]$. Recently, the notion of rough group, rough subgroup and some properties were studied [1]. Groupoid was introduced by Brandt [11] on the composition of quadratic forms with four variables. Grothendick [12] used groupoid for the construction of module space. Also groupoids play an essential role in physics and mathematics as moduli space. In algebraic topology, the fundamental groupoid of a topological space has been exploited by R.Brown and other $[13,14,15,16,17,18,19,20,21,22,23,24]$.

Groupoid have been used in a wide different area of mathematic such as functional analysis, ergodic theory, algebraic topology, algebraic geometry, differential geometry and group theory.

In this article we define the concept of rough subgroupoid of a groupoid and discuss some features about it.

Received February 11, 2019 - Accepted April 7, 2019.

(C) The Author(s) 2017. This article is published with open access by Sidi Mohamed Ben Abdallah University.

${ }^{1}$ Department of Mathematics, Hatay Mustafa Kemal University, Hatay, TURKEY.

${ }^{2}$ Department of Mathematics, Inn University, Malatya, TURKEY.

a e-mail: htasbozan@mku.edu.tr

b e-mail: ilhan.icen@inonu.edu.tr . 


\section{Preliminaries}

Definition 2.1. Let $U$ be a nonempty set and let $R$ be an equivalence relation on $U$. Then $S=(U, R)$ is called the approximation space and $U$ is called a universe [2].

Definition 2.2. Let $S=(U, R)$ be an approximation space. Suppose $\varnothing \neq X \subseteq U$. Then the sets

$$
\begin{aligned}
& \bar{R}(X)=\left\{x \mid[x]_{R} \cap X \neq \varnothing\right\} \\
& \underline{R}(X)=\left\{x \mid[x]_{R} \subseteq X\right\}
\end{aligned}
$$

are called respectively lower and upper approximations of the set $X$ in the approximation space $S$, where $[x]_{R}$ denotes the equivalence class of the relation $R$ containing $x$. The set $R(X)=(\underline{R}(X), \bar{R}(X))$ is called the rough set of $X$ in $S$. The set $\bar{R}(X)-\underline{R}(X)$ is called the boundary region of $X$. If the boundary region of $X$ is not an empty set, then $X$ is a rough set.

For a fixed approximation space $S=(U, R)$ and for a fixed nonempty subset $X$ of $U$, the rough set of $X$, i.e. $R(X)$ is unique [2].

Definition 2.3. A groupoid $G$ consists of two sets $G$ and $G_{0}$ called respectively the set of elements or morphisms and the set of objects of the groupoid, together with two maps $\alpha, \beta: G \rightarrow G_{0}$, called respectively the source and target maps, a map $1_{()}: G_{0} \rightarrow G, x \mapsto 1_{x}$ called the object map and a partial multiplication

$$
G \times G \rightarrow G,(a, b) \mapsto a \circ b
$$

defined on the fibre product set

$$
G \times G=\{(a, b) \in G \times G: \alpha(b)=\beta(a)\} .
$$

These maps are subject to the following conditions:

(1) $\alpha(b \circ a)=\alpha(a)$ and $\beta(b \circ a)=\beta(b)$ for all $a, b \in G$;

(2) $c \circ(b \circ a)=(c \circ b) \circ$ a for all $a, b, c \in G$ such that $\alpha(c)=\beta(b)$ and $\alpha(b)=\beta(a)$;

(3) $\alpha\left(1_{x}\right)=\beta\left(1_{x}\right)=x$ for all $x \in G_{0}$, where $1_{x}$ is the identity at $x$;

(4) $a \circ 1_{\alpha(a)}=a$ and $1_{\beta(a)} \circ a=a$ for all $a \in G$;

(5) each $a \in G$ has an inverse $a^{-1}$ such that $\alpha\left(a^{-1}\right)=\beta(a), \alpha(a)=\beta\left(a^{-1}\right)$ and $a^{-1} \circ a=1_{\alpha(a)}, a \circ a^{-1}=1_{\beta(a)}$

If $\left(G, G_{0}\right)$ is a groupoid then we say $G$ is a groupoid on $G_{0}[13]$.

Definition 2.4. Let $G$ be a groupoid on $G_{0}$. A subgroupoid $H$ of $G$ is a pair of subsets $H \subseteq G$ and $H_{0} \subseteq G_{0}$ such that $\alpha(H) \subseteq H_{0}, \beta(H) \subseteq H_{0}, 1_{x} \in H$ for $x \in H_{0}$ and $H$ is closed under the partial multiplication and inversion in $G$ [13].

Definition 2.5. A normal subgroupoid $N$ of $G$ is subgroupoid $N$ of $G$ such that $N_{0}=G_{0}$ and for each $x, y, z \in G_{0}, a \in$ $G(z, y), b \in G(x, z), a \circ b \in G(x, z)$ and for $\alpha(a)=\beta(b)$ we have $a \circ N(z)=N(y) \circ a$ and $(a \circ N(z)) \circ(b \circ N(x))=a$ $\circ b \circ N(x)$. In other words, a subgroupoid $N$ of $G$ is called normal if $N_{0}=G_{0}$ and for $x, y \in G_{0}$ and $a \circ N(z) \circ a^{-1}=N(y)$ $[13,25]$.

\section{Lower and Upper Approximations in a Groupoid}

Definition 3.1. Let $G$ be a groupoid, $N$ be a normal subgroupoid of $G$ and $N_{x}: x \rightarrow x$ be a morphism. Let $A$ be a nonempty subset of $G$ and $A_{0}$ is the set of end points of the morphisms in $A$. Then the sets

$$
\begin{aligned}
\underline{N_{x}}(A) & =\left\{a \in G \mid a \circ N_{x} \subseteq A, \alpha(a), \beta(a) \in A_{0}, x \in G_{0}\right\}, \\
\underline{N}(A) & =\bigcup_{x \in G_{0}} \underline{N_{x}}(A)
\end{aligned}
$$

and

$$
\begin{aligned}
\overline{N_{x}}(A) & =\left\{a \in G \mid a \circ N_{x} \cap A \neq \varnothing, \alpha(a), \beta(a) \in A_{0}, x \in G_{0}\right\} \\
\bar{N}(A) & =\bigcup_{x \in G_{0}} \overline{N_{x}}(A)
\end{aligned}
$$


are called respectively, lower and upper approximations of a set $A$ with respect to the normal subgroupoid $N$ of G. Then we say that the pair of $N(A)=\left(\cup_{x \in G_{0}} \frac{N_{x}}{}(A), \underset{x \in G_{0}}{\cup} \overline{N_{x}}(A)\right)$ is a rough set of $A$ in $G$.

Definition 3.2. Let $N(A)=(\underline{N}(A), \bar{N}(A))$ be a rough set of $A$ in $G$. A nonempty subset $A$ of a groupoid $G$ is called an $\bar{N}$ rough(normal) subgroupoid of $G$ if the upper approximation $\underset{x \in G_{0}}{\cup} \bar{N}(A)$ of $A$ is a (normal)subgroupoid of $G$. Similarly a nonempty subset $A$ of $G$ is called an $\underline{N}$ rough (normal) subgroupoid of $G$ if the lower approximation $\underset{x \in G_{0}}{\cup}(A)$ of $A$ is a (normal)subgroupoid of $G$.

Example 3.1. $G=\left\{1_{0}, a, a^{-1}, 1_{1}\right\}$ is a groupoid with $a: 0 \rightarrow 1, a^{-1}: 1 \rightarrow 0$ and $N=\left\{1_{0}, 1_{1}\right\}$ is a normal subgroupoid of $G$. Let us take $N_{0}=G_{0}, N_{0}$ is the set of the morphism with source objects of $G$ and the set $A=\{a\} \subseteq G$. In this case, we have $a \circ N_{0}=\{a\} \subseteq A$ and $a^{-1} \circ N_{0}=\left\{a^{-1}\right\}$. Then, we have

and also

$$
\begin{gathered}
\underline{N_{0}}(A)=\left\{a \in G \mid a \circ N_{0} \subseteq A\right\}=A, \\
\overline{N_{0}}(A)=\left\{a \in G \mid a \circ N_{0} \cap A \neq \varnothing\right\}=A
\end{gathered}
$$

$$
\begin{aligned}
& \underline{N}(A)=\cup_{x \in G_{0}} \underline{N_{x}}(A)=A, \\
& \bar{N}(A)=\cup_{x \in G_{0}} \overline{N_{x}}(A)=A .
\end{aligned}
$$

Because of $\bar{N}(A)-\underline{N}(A)=\varnothing, A$ is not a $\bar{N}$ rough subgroupoid of $G$ and a $\underline{N}$ rough subgroupoid of $G$.

Example 3.2. Let $X=\{x, y, z\}$ be a set. Then there is a groupoid with object set $X$ and set of arrows the product set $X \times X$ so that an arrow $x \rightarrow y$ is simply the ordered pair $(y, x)$. The composition is then given by $(z, y) \circ(y, x)=(z, x)$. Then $R=X \times X$ is an equivalence relation on $X . R$ is a groupoid with the composition [14].

$$
R=\{(x, x),(y, y),(z, z),(x, y),(y, x),(x, z),(z, x),(y, z),(z, y)\}
$$

is a groupoid,

is a normal subgroupoid of $N$ and let

$$
N=\{(x, x),(y, y),(z, z)\}
$$

$$
A=\{(x, x),(y, y),(y, x),(z, x),(y, z)\} \subset R
$$

be a set. We have

$$
\begin{aligned}
a \circ N_{x} & =\{(x, x),(x, y),(x, z)\}, \\
a \circ N_{y} & =\{(y, y),(y, x),(y, z)\}, \\
a \circ N_{z} & =\{(z, z),(z, x),(z, y)\},
\end{aligned}
$$

and thus from the Definition 6, we obtain

$$
\begin{aligned}
& \frac{N_{x}}{N_{x}}(A)=\varnothing, \\
& \frac{N_{z}}{N_{z}}(A)=\varnothing, \\
& \underline{N}(A)=\underline{N_{x}}(A) \cup \overline{N_{y}}(A) \cup \underline{N_{z}}(A)=\{(y, y),(y, x),(y, z)\}, \\
& \bar{N}(A)=\overline{\overline{N_{x}}}(A) \cup \overline{\overline{N_{y}}}(A) \cup \overline{\overline{N_{z}}}(A)=R .
\end{aligned}
$$

$A$ is a $\bar{N}$ rough subgroupoid of $G$ and $\underline{N}$ rough subgroupoid of $G$.

Proposition 3.1. Let $N$ and $H$ be normal subgroupoids of a groupoid $G$. Let $A$ and $B$ be any nonempty subsets of $G$. Then

(1) $\underset{x \in G_{0}}{\cup} \underline{N_{x}}(A) \subseteq A \subseteq \underset{x \in G_{0}}{\cup} \overline{N_{x}}(A)$.

(2) $\underset{x \in G_{0}}{\cup} \overline{N_{x}}(A \cup B)=\left(\underset{x \in G_{0}}{\cup} \overline{N_{x}}(A)\right) \cup\left(\cup_{x \in G_{0}} \overline{N_{x}}(B)\right)$. 
(3) $\underset{x \in G_{0}}{\cup} \underline{N_{x}}(A \cap B)=\left(\underset{x \in G_{0}}{\cup} \underline{N_{x}}(A)\right) \cap\left(\cup_{x \in G_{0}} \underline{N_{x}}(B)\right)$.

(4) $A \subseteq B$ implies $\underset{x \in G_{0}}{\cup} \frac{N_{x}}{N_{x}}(A) \subseteq \underset{x \in G_{0}}{\cup} \frac{N_{x}}{(B)}$.

(5) $A \subseteq B$ implies $\bigcup_{x \in G_{0}} \overline{N_{x}}(A) \subseteq \bigcup_{x \in G_{0}} \overline{N_{x}}(B)$.

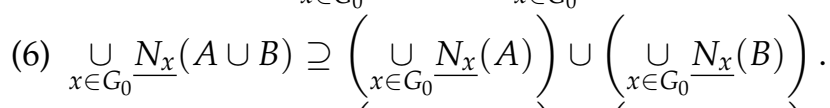

(7) $\underset{x \in G_{0}}{\cup} \overline{N_{x}}(A \cap B) \subseteq\left(\cup_{x \in G_{0}} \overline{N_{x}}(A)\right) \cap\left(\cup_{x \in G_{0}} \overline{N_{x}}(B)\right)$.

(8) $N \subseteq H$ implies $\underset{x \in G_{0}}{\cup} \frac{N_{x}}{}(A) \subseteq \underset{x \in G_{0}}{\cup} \underline{H_{x}}(A)$.

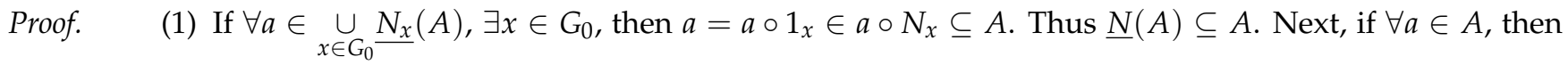
$a=a \circ 1_{x} \in a \circ N_{x}$. Thus $a \in a \circ N_{x} \cap A$, that is, $\forall x \in G_{0}, a \circ N_{x} \cap A \neq \varnothing$. This implies $a \in \underset{x \in G_{0}}{\cup} \overline{N_{x}}(A)$, and so $A \subseteq \cup_{x \in G_{0}} \overline{N_{x}}(A)$.

(2) $a \in \underset{x \in G_{0}}{\cup} \overline{N_{x}}(A \cup B) \Leftrightarrow a \circ N_{x} \cap(A \cup B) \neq \varnothing, \forall x \in G_{0}$

$$
\begin{aligned}
& \Leftrightarrow\left(a \circ N_{x} \cap A\right) \cup\left(a \circ N_{x} \cap B\right) \neq \varnothing, \forall x \in G_{0} \\
\Leftrightarrow & \left(a \circ N_{x} \cap A\right) \neq \varnothing \text { or }\left(a \circ N_{x} \cap B\right) \neq \varnothing \\
\Leftrightarrow & a \in \underset{x \in G_{0}}{\cup} \overline{N_{x}}(A) \text { or } a \in \underset{x \in G_{0}}{\cup} \overline{N_{x}}(B) \\
\Leftrightarrow & a \in\left(\cup_{x \in G_{0}} \overline{N_{x}}(A)\right) \cup\left(\cup_{x \in G_{0}} \overline{N_{x}}(B)\right)
\end{aligned}
$$

Thus $\underset{x \in G_{0}}{\cup} \overline{N_{x}}(A \cup B)=\left(\underset{x \in G_{0}}{\cup} \overline{N_{x}}(A)\right) \cup\left(\underset{x \in G_{0}}{\cup} \overline{N_{x}}(B)\right)$.

(3) $a \in \underset{x \in G_{0}}{\cup} N_{x}(A \cap B) \Leftrightarrow a \circ N_{x} \subseteq A \cap B, \forall x \in G_{0}$

$$
\begin{aligned}
& \Leftrightarrow a \circ N_{x} \subseteq A \text { and } a \circ N_{x} \subseteq B \\
& \Leftrightarrow a \in \bigcup_{x \in G_{0}} \underline{N_{x}}(A) \text { and } a \in \bigcup_{x \in G_{0}} \underline{N_{x}}(B) \\
& \Leftrightarrow a \in\left(\bigcup_{x \in G_{0}} \underline{N_{x}}(A)\right) \cap\left(\bigcup_{x \in G_{0}} \underline{N_{x}}(B)\right)
\end{aligned}
$$

Thus $\quad \underset{x \in G_{0}}{\cup} \underline{N_{x}}(A \cap B)=\left(\underset{x \in G_{0}}{\cup} \underline{N_{x}}(A)\right) \cap\left(\cup_{x \in G_{0}} \underline{N_{x}}(B)\right)$.

(4) Since $A \subseteq B, A \cap B=A$. With the help of (3),

$$
\cup \in G_{0} \underline{N_{x}}(A)=\cup_{x \in G_{0}} \underline{N_{x}}(A \cap B)=\left(\cup_{x \in G_{0}} \underline{N_{x}}(A)\right) \cap\left(\cup_{x \in G_{0}} \underline{N_{x}}(B)\right)
$$

gives us $\underset{x \in G_{0}}{\cup} \underline{N_{x}}(A) \subseteq \underset{x \in G_{0}}{\cup} \underline{N_{x}}(B)$.

(5) Since $A \subseteq B, A U B=B$. With the help of (2),

$$
\bigcup_{x \in G_{0}} \overline{N_{x}}(A)=\cup_{x \in G_{0}} \overline{N_{x}}(A \cup B)=\left(\cup_{x \in G_{0}} \overline{N_{x}}(A)\right) \cup\left(\underset{x \in G_{0}}{\cup} \overline{N_{x}}(A)(B)\right)
$$

gives us $\underset{x \in G_{0}}{\cup} \overline{N_{x}}(A) \subseteq \underset{x \in G_{0}}{\cup} \overline{N_{x}}(B)$.

(6) $A \subseteq A \cup B, B \subseteq A \cup B$ and with the help of (4), $\underset{x \in G_{0}}{\cup} \frac{N_{x}}{(A) \subseteq} \underset{x \in G_{0}}{\cup} \frac{N_{x}}{}(A \cup B)$ and $\underset{x \in G_{0}}{\cup} \frac{N_{x}}{}(B) \subseteq$ $\cup \cup_{x \in G_{0}} \underline{N_{x}}(A \cup B)$. Therefore

$$
\left(\cup_{x \in G_{0}} \underline{N_{x}}(A)\right) \cup\left(\cup_{x \in G_{0}} \underline{N_{x}}(B)\right) \subseteq \cup_{x \in G_{0}} \underline{N_{x}}(A \cup B) .
$$


(7) $A \cap B \subseteq A, A \cap B \subseteq B$ and with the help of (5), $\underset{x \in G_{0}}{\cup} \overline{N_{x}}(A \cap B) \subseteq \underset{x \in G_{0}}{\cup \overline{N_{x}}}(A)$ and $\underset{x \in G_{0}}{\cup} \overline{N_{x}}(A \cap B) \subseteq$ $\cup_{x \in G_{0}} \overline{N_{x}}(B)$. Therefore

$$
\bigcup_{x \in G_{0}} \overline{N_{x}}(A \cap B) \subseteq\left(\cup_{x \in G_{0}} \overline{N_{x}}(A)\right) \cap\left(\bigcup_{x \in G_{0}} \overline{N_{x}}(B)\right) .
$$

(8) If $\forall c \in \underset{x \in G_{0}}{\cup} \overline{N_{x}}(A)$, then $\forall x \in G_{0}, \exists x \in c \circ N_{x} \cap A$, and so $x \in c \circ N_{x} \subseteq c \circ H_{x}$. Therefore, $x \in c \circ H_{x} \cap$ $A$. This gives us $c \in \underset{x \in G_{0}}{\cup} \overline{H_{x}}(A)$, and $\underset{x \in G_{0}}{\cup} \overline{N_{x}}(A) \subseteq \underset{x \in G_{0}}{\cup} \overline{H_{x}}(A)$.

Proposition 3.2. Let $N$ be a normal subgroupoid of a groupoid $G$. Let $\varnothing \neq A \subseteq G$ and $\varnothing \neq B \subseteq G$ are subgroupoids of G. Then $\left(\underset{x \in G_{0}}{\cup} \overline{N_{x}}(A)\right) \circ\left(\cup_{x \in G_{0}} \overline{N_{x}}(B)\right)=\cup_{x \in G_{0}} \overline{N_{x}}(A \circ B)$.

Proof. Let $N$ be a normal subgroupoid of a groupoid $G$ such that $N_{0}=G_{0}$ and for each $x, y, z \in G_{0}, a \in G(z, y), b \in$ $G(x, z), a \circ b \in G(x, z)$ and for $\alpha(b)=\beta(a)$ we have $(a \circ N(z)) \circ(b \circ N(x))=a \circ b \circ N(x)$. Let $c \in \underset{x \in G_{0}}{\cup} \overline{N_{x}}(A \circ B)$. Then $\forall x \in G_{0}, c \circ N_{x} \cap A \circ B \neq \varnothing$. Thus there exists an element $m$ in $G$ such that $m \in c \circ N_{x} \cap A \circ B$, and so $m \in c \circ N_{x}$ and $m \in c \circ A \circ B$. Then $m=a \circ b$ with $a \in A$ and $b \in B$. Since $c \in m \circ N_{x}=(a \circ b) \circ$ $N_{x}=\left(a \circ N_{z}\right) \circ\left(b \circ N_{x}\right)$, we have $c=k \circ l$ with $k \in a \circ N_{z}$ and $l \in b \circ N_{x}$. Then $a \in k \circ N_{z}$, and so $a \in k \circ N_{z}$ $\cap A$. Thus $k \in \underset{x \in G_{0}}{\cup} \overline{N_{x}}(A)$. We have same $l \in \underset{x \in G_{0}}{\cup} \overline{N_{x}}(B)$. Then $c=k \circ l \in\left(\underset{x \in G_{0}}{\cup} \overline{N_{x}}(A)\right) \circ\left({ }_{x \in G_{0}} \overline{N_{x}}(B)\right)$, gives $\cup_{x \in G_{0}} \overline{N_{x}}(A \circ B) \subseteq\left(\cup_{x \in G_{0}} \overline{N_{x}}(A)\right) \circ\left(\cup_{x \in G_{0}} \overline{N_{x}}(B)\right)$. Contrarily, let $c \in\left(\underset{x \in G_{0}}{\cup} \overline{N_{x}}(A)\right) \circ\left(\underset{x \in G_{0}}{\cup} \overline{N_{x}}(B)\right)$, then $c=a \circ b$ with $a \in \underset{x \in G_{0}}{\cup} \overline{N_{x}}(A)$ and $b \in \underset{x \in G_{0}}{\cup} \overline{N_{x}}(A)$. Then there consists $r, s \in G$ with $r \in a \circ N_{z} \cap A$ and $s \in b \circ N_{x} \cap B$, and so $r \in a \circ N_{z}, r \in A, s \in b \circ N_{x}, s \in B$. Since $N$ is normal, $r \circ s \in\left(a \circ N_{z}\right)\left(b \circ N_{x}\right)=a \circ b \circ N_{x}$, and $r \circ s \in A \circ B$. Thus $r \circ s \in a \circ b \circ N_{x} \cap A \circ B$, which yields that $c=a \circ b \in \underset{x \in G_{0}}{\cup} \overline{N_{x}}(A \circ B)$, and so $\left(\underset{x \in G_{0}}{\cup} \overline{N_{x}}(A)\right) \circ\left(\cup_{x \in G_{0}} \overline{N_{x}}(A)\right) \subseteq$ $\bigcup_{x \in G_{0}} \overline{N_{x}}(A \circ B)$. For this reason we have $\left(\underset{x \in G_{0}}{\cup} \overline{N_{x}}(A)\right) \circ\left(\underset{x \in G_{0}}{\cup} \overline{N_{x}}(B)\right)=\underset{x \in G_{0}}{\cup} \overline{N_{x}}(A \circ B)$.

Proposition 3.3. Let $N$ be a normal subgroupoid of a groupoid $G, \varnothing \neq A \subseteq G$ and $\varnothing \neq B \subseteq G$ are subgroupoids of $G$. Then $\left(\underset{x \in G_{0}}{\cup} \underline{N_{x}}(A)\right) \circ\left(\underset{x \in G_{0}}{\cup} \underline{N_{x}}(B)\right) \subseteq \underset{x \in G_{0}}{\cup} \underline{N_{x}}(A \circ B)$.

Proof. Let $N$ be a normal subgroupoid of a groupoid $G$ such that $N_{0}=G_{0}$ and for each $x, y, z \in G_{0}, a \in G(z, y), b \in$ $G(x, z), a \circ b \in G(x, z)$ and for $\alpha(b)=\beta(a)$ we have $(a \circ N(z)) \circ(b \circ N(x))=a \circ b \circ N(x)$. Let $c \in\left(\cup_{x \in G_{0}} \underline{N_{x}}(A)\right) \circ$ $\left(\cup_{x \in G_{0}} \frac{N_{x}}{(B)}\right)$. Then $a \in \underset{x \in G_{0}}{\cup} \underline{N_{x}}(A)$ and $b \in \underset{x \in G_{0}}{\cup} \underline{N_{x}}(B)$ gives $c=a \circ b$. Then $a \circ N_{z} \subseteq A$ and $b \circ N_{x} \subseteq B$. Since $N$ is normal, $c \circ N_{x}=a \circ b \circ N_{x}=\left(a \circ N_{z}\right) \circ\left(b \circ N_{x}\right) \subseteq A \circ B$, then $c \in \underset{x \in G_{0}}{\cup} \frac{N_{x}}{}(A \circ B)$. Therefore $\left(\cup_{x \in G_{0}} \underline{N_{x}}(A)\right) \circ\left(\cup_{x \in G_{0}} \underline{N_{x}}(B)\right) \subseteq \cup_{x \in G_{0}} \underline{N_{x}}(A \circ B)$.

Remark 3.1. Let $H$ and $N$ be normal subgroupoids of a groupoid $G$. Clearly $H \cap N$ is also a normal subgroupoid of $G$.

Proposition 3.4. Let $H$ and $N$ be normal subgroupoids of a groupoid $G$ and $\varnothing \neq A \subseteq G$. Then $\underset{x \in G_{0}}{\cup} \overline{(H \cap N)_{x}}(A)=$ $\left(\cup_{x \in G_{0}} \overline{H_{x}}(A)\right) \cap\left(\cup_{x \in G_{0}} \overline{N_{x}}(A)\right)$. 
Proof. Let $c \in \underset{x \in G_{0}}{\cup} \overline{(H \cap N)_{x}}(A)$. Then $c \circ(H \cap N)_{x} \cap A \neq \varnothing$. This gives $a \in c \circ(H \cap N)_{x} \cap A, \forall x \in G_{0}$. Since $a \in c \circ(H \cap N)_{x}$ and $a \in A$, then $a \in c \circ H_{x}, a \in A$ and $a \in c \circ N_{x}, a \in A, \forall x \in G_{0}$. Thus $a \in c \circ H_{x} \cap A$ and $\exists a \in c \circ N_{x} \cap A, \forall x \in G_{0}$. Therefore $c \in \underset{x \in G_{0}}{\cup} \overline{H_{x}}(A)$ and $c \in \underset{x \in G_{0}}{\cup} \overline{N_{x}}(A)$.

$$
\text { Thus } \underset{x \in G_{0}}{\cup} \overline{(H \cap N)_{x}}(A)=\left(\cup_{x \in G_{0}} \overline{H_{x}}(A)\right) \cap\left(\cup_{x \in G_{0}} \overline{N_{x}}(A)\right) \text {. }
$$

Proposition 3.5. Let $H$ and $N$ be normal subgroupoids of a groupoid $G$. Let $A$ be a nonempty subset of $G$. Then $\bigcup_{x \in G_{0}} \underline{(H \cap N)_{x}}(A)=\left(\cup_{x \in G_{0}} \underline{H_{x}}(A)\right) \cap\left(\cup_{x \in G_{0}} \underline{N_{x}}(A)\right)$.

Proof. $\forall c \in \underset{x \in G_{0}}{\cup} \underline{(H \cap N)_{x}}(A) \Leftrightarrow c \circ(H \cap N)_{x} \subseteq A$

$$
\begin{aligned}
& \Leftrightarrow c \circ H_{x} \subseteq A \text { and } c \circ N_{x} \subseteq A, \forall x \in G_{0} \\
& \Leftrightarrow c \in \underset{x \in G_{0}}{\cup} \underline{H_{x}}(A) \text { and } c \in \cup_{x \in G_{0}} \underline{N_{x}}(A) \\
& \Leftrightarrow c \in\left(\bigcup_{x \in G_{0}} \underline{H_{x}}(A)\right) \cap\left(\bigcup_{x \in G_{0}} \underline{N_{x}}(A)\right) \\
& \text { Thus } \cup_{x \in G_{0}} \underline{(H \cap N)_{x}}(A)=\left(\bigcup_{x \in G_{0}} \underline{H_{x}}(A)\right) \cap\left(\bigcup_{x \in G_{0}} \underline{N_{x}}(A)\right) .
\end{aligned}
$$

Proposition 3.6. Let $N$ be a normal subgroupoid of a groupoid $G$. If $A$ is a subgroupoid of $G$, then it is an $\underset{x \in G_{0}}{\cup} \overline{N_{x}}$ rough subgroupoid of $G$.

Proof. Let $N$ be a normal subgroupoid of a groupoid $G$ such that $N_{0}=G_{0}$ and for each $x, y, z \in G_{0}, a \in G(z, y)$, $b \in G(x, z), a \circ b \in G(x, z)$ and for $\alpha(b)=\beta(a)$ we have $(a \circ N(z)) \circ(b \circ N(x))=a \circ b \circ N(x)$. Let $1_{x}$ be the identity of $\mathrm{G}$. Since $\mathrm{N}$ and $\mathrm{A}$ are subgroups of $\mathrm{G}, 1_{x} \in \mathrm{A}$ and $1_{x}=1_{x} \circ 1_{x} \in 1_{x} \circ N_{x}$, and so $1_{x} \in 1_{x} \circ N_{x} \cap A$. Thus $1_{x} \circ N_{x} \cap A \neq \varnothing$. This implies that $1_{x} \in \underset{x \in G_{0}}{\cup} \overline{N_{x}}(A), \forall x \in G_{0}$. Let $a, b \in \underset{x \in G_{0}}{\cup \overline{N_{x}}}(A)$. Then $k, l \in G$ so $k \in a \circ N_{z} \cap A$ and $l \in b \circ N_{x} \cap A$. Thus $k \in a \circ N_{z}, l \in b \circ N_{x}$ and $k \in A, l \in A$. Since $A$ is a subgroupoid of $G, k \circ l \in A$. And since $N$ is a normal subgroupoid of $G, k \circ l \in\left(a \circ N_{z}\right) \circ\left(b \circ N_{x}\right)=a \circ b \circ N_{x}$. Thus $k \circ l \in$ $a \circ b \circ N_{x} \cap A$, and so $a \circ b \in \underset{x \in G_{0}}{\cup} \overline{N_{x}}(A)$. Let $a$ be any element of $\underset{x \in G_{0}}{\cup} \overline{N_{x}}(A)$. Then $k \in a \circ N_{z} \cap A$ for some $k \in G$, that is, $k \in a \circ N_{z}$ and $k \in A$. Then since $A$ is a subgroupoid of $G, k^{-1} \in A$. On the other hand, since $k=a \circ h$ for some $h \in N_{z}$, and since $N$ is a normal subgroupoid of $G, h^{-1} \in N_{z}$ and it implies that $k^{-1}=(a \circ h)^{-1}=h^{-1} \circ a^{-1} \in N_{z} \circ a^{-1}=a^{-1} \circ N_{y}$. Thus $k^{-1} \in a^{-1} \circ N_{y} \cap A$, and so $a^{-1} \in \underset{x \in G_{0}}{\cup} \overline{N_{x}}(A)$. This show that $\underset{x \in G_{0}}{\cup} \overline{N_{x}}(A)$ is a subgroupoid of $G$.

Proposition 3.7. Let $N$ be a normal subgroupoid of a groupoid $G$. If $A$ is a normal subgroupoid of $G$, then it is an $\underset{x \in G_{0}}{\cup} \overline{N_{x}}$ rough normal subgroupoid of $G$.

Proof. We must indicate $\underset{x \in G_{0}}{\cup} \overline{N_{x}}(A)$ is normal. Let $a \in G(x, x) \in \underset{x \in G_{0}}{\cup} \overline{N_{x}}(A)$ and $k \in G(x, y) \in G$ for each $x, y \in G_{0}$. Then $l \in G$ such that $l \in a \circ N_{x} \cap A$, that is, $l \in a \circ N_{x}$ and $l \in A$. Since $N$ is normal, $k \circ l \circ k^{-1}$ $\in k \circ\left(a \circ N_{x}\right) \circ k^{-1}=(k \circ a) \circ\left(N \circ k^{-1}\right)=(k \circ a) \circ\left(k^{-1} \circ N_{y}\right)=\left(k \circ a \circ k^{-1}\right) \circ N_{y}$. Since $A$ is normal, $k \circ l \circ k^{-1} \in$ $k A k^{-1} \subseteq A$. Thus $k \circ l \circ k^{-1} \in\left(k \circ a \circ k^{-1}\right) \circ N_{y} \cap A$, and so $k \circ a \circ k^{-1} \in \underset{x \in G_{0}}{\cup} \overline{N_{x}}(A)$. This implies that $\underset{x \in G_{0}}{\cup} \overline{N_{x}}(A)$ is normal.

Proposition 3.8. Let $N$ be a normal subgroupoid of a groupoid $G$. If $A$ is a subgroupoid of $G$ which $N \subseteq A$, then it is an $\cup \mathrm{N}_{x \in \mathrm{N}_{0}}$ rough subgroupoid of $\mathrm{G}$.

Proof. Let $N$ be a normal subgroupoid of a groupoid $G$ such that $N_{0}=G_{0}$ and for each $x, y, z \in G_{0}, a \in G(z, y)$ $, b \in G(x, z), a \circ b \in G(x, z)$ and for $\alpha(b)=\beta(a)$ we have $(a \circ N(z)) \circ(b \circ N(x))=a \circ b \circ N(x)$. Since $A$ and 
$N$ are subgroupoids of $G, 1_{x} \in A, 1_{x} \in N_{x}$. Since $1_{z} \circ N_{z}=N_{z} \subseteq A, 1_{z} \in \underset{x \in G_{0}}{\cup} \frac{N_{x}}{}(A)$ and $1_{x} \circ N_{x}=N_{x} \subseteq A$, then $1_{z} \in \underline{N}(A)$ and $1_{x} \in \underset{x \in G_{0}}{\cup} \underline{N_{x}}(A)$. Let $a, b \in \underset{x \in G_{0}}{\cup} \underline{N_{x}}(A)$. Then $a \circ N_{z} \subseteq A$ and $b \circ N_{x} \subseteq A$. SinceN is a normal subgroupoid and $A$ is a subgroupoid of $G, a \circ b \circ N_{x}=\left(a \circ N_{z}\right) \circ\left(b \circ N_{x}\right) \subseteq A \circ A \subseteq A$. So that $a \circ b \in \underset{x \in G_{0}}{\cup} N_{x}(A)$. Let $a \in \underset{x \in G_{0}}{\cup} \underline{N_{x}}(A)$. Then $a=a \circ 1_{z} \in a \circ N_{z} \subseteq A$. Since $A$ is a subgroupoid of $G, a^{-1} \in A$.

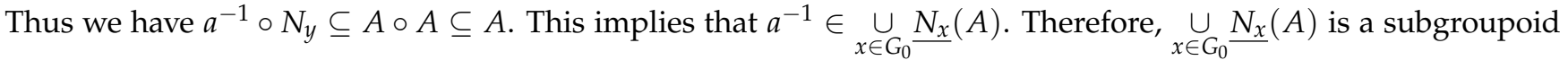
of $G$.

Proposition 3.9. Let $N$ be a normal subgroupoid of a groupoid $G$. If $A$ is a normal subgroupoid of $G$ such that $N \subseteq A$, then it is an $\underset{x \in G_{0}}{\cup} \underline{N_{x}}(A)$ rough normal subgroupoid of $G$.

Proof. We must indicate $\underset{x \in G_{0}}{\cup} \frac{N_{x}}{(A)}$ is normal. Let $a \in G(x, x) \underset{x \in G_{0}}{\cup} \underline{N_{x}}(A)$ and $k \in G(x, y)$ for each $x, y \in G_{0}$. Then, $a \circ N_{x} \subseteq A$. Since $N$ and $A$ are normal,

$$
(k \circ a \circ k)^{-l} \circ N_{x}=k \circ\left(a \circ N_{x}\right) \circ k^{-1} \subseteq k \circ A \circ k^{-1} \subseteq A
$$

and so $k \circ A \circ k^{-1} \in \underset{x \in G_{0}}{\cup} \underline{N_{x}}(A)$. Thus $\underset{x \in G_{0}}{\cup} \underline{N_{x}}(A)$ is normal.

Proposition 3.10. Let $H$ and $N$ be normal subgroupoids of a groupoid $G$. If $A$ is a subgroupoid of $G$, then $\left(\underset{x \in G_{0}}{\cup} \overline{H_{x}}(A)\right)$ $\circ\left(\cup_{x \in G_{0}} \overline{N_{x}}(A)\right) \subseteq \cup_{x \in G_{0}} \overline{(H \circ N)_{x}}(A)$.

Proof. Let $N$ be a normal subgroupoid of a groupoid $G$ such that $N_{0}=G_{0}$ and for each $x, y, z \in G_{0}, a \in G(z, y)$, $b \in G(x, z), a \circ b \in G(x, z)$ and for $\alpha(b)=\beta(a)$ we have $(a \circ N(z)) \circ(b \circ N(x))=a \circ b \circ N(x)$. Let $c \in \underset{x \in G_{0}}{\cup} \overline{H_{x}}(A)$ $\circ \underset{x \in G_{0}}{\cup} \overline{N_{x}}(A)$. Then $c=a \circ b$ with $a \in \underset{x \in G_{0}}{\cup} \overline{H_{x}}(A)$ and $b \in \underset{x \in G_{0}}{\cup} \overline{N_{x}}(A)$. Then $k, l \in G$ which $k \in a \circ H_{z} \cap A$ and $l \in b \circ N_{x} \cap A$. Thus $\mathrm{k} \in a \circ H_{z}, l \in b \circ N_{x}, k \in A, l \in A$. Then, since $H$ is normal,

$$
\begin{aligned}
& k \circ l \in\left(a \circ H_{z}\right) \circ\left(b \circ N_{x}\right)=\left\{a \circ\left(H_{z} \circ b\right) \circ N_{x}\right\}=\left\{a \circ\left(b \circ H_{x}\right) \circ N_{x}\right\}=\left\{(a \circ b) \circ H_{x}\right\} \circ N_{x} \\
& =(a \circ b) \circ H_{x} \circ N_{x}=c \circ H_{x} \circ N_{x} .
\end{aligned}
$$

Since $A$ is a subgroupoid of $G, k \circ l \in A$. Therefore, $k \circ l \in c \circ H_{x} \circ N_{x} \cap A$, and so we have $c \in \underset{x \in G_{0}}{\cup} \overline{(H \circ N)_{x}}(A)$. Thus $\left(\underset{x \in G_{0}}{\cup} \overline{H_{x}}(A)\right) \circ\left(\cup_{x \in G_{0}} \overline{N_{x}}(A)\right) \subseteq \underset{x \in G_{0}}{\cup} \overline{(H \circ N)_{x}}(A)$. This completes the proof.

Proposition 3.11. Let $H$ and $N$ be normal subgroupoids of a groupoid $G$. If $A$ is a subgroupoid of $G$, then $\left(\underset{x \in G_{0}}{\cup} \underline{H}_{x}(A)\right)$ $\circ\left(\cup_{x \in G_{0}} \underline{N_{x}}(A)\right) \subseteq \underset{x \in G_{0}}{\cup} \underline{(H \circ N)_{x}}(A)$.

Proof. Let $N$ be a normal subgroupoid of a groupoid $G$ such that $N_{0}=G_{0}$ and for each $x, y, z \in G_{0}, a \in$ $G(z, y), b \in G(x, z), a \circ b \in G(x, z)$ and for $\alpha(b)=\beta(a)$ we have $(a \circ N(z)) \circ(b \circ N(x))=a \circ b \circ N(x)$. Let $c \in$

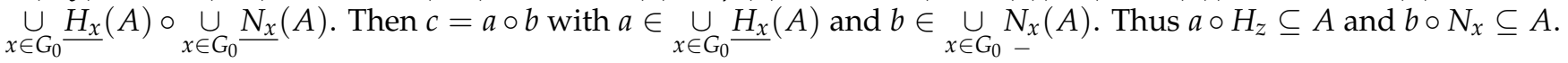
Since $H$ is a normal subgroupoid and $A$ is a subgroupoid of $G$, we have $(a \circ b) \circ H_{x} \circ N_{x}=\left\{a \circ\left(b \circ H_{x}\right)\right\} \circ N_{x}=$ $\left\{a \circ\left(H_{z} \circ b\right)\right\} \circ N_{x}=\left\{\left(a \circ H_{z}\right) \circ b\right\} \circ N_{x}=\left(a \circ H_{z}\right) \circ\left(b \circ N_{x}\right) \subseteq A \circ A \subseteq A$. Therefore, we have $c=a \circ b \in$ $\cup \underset{x \in G_{0}}{\cup} \underline{(H \circ N)_{x}}(A)$, and so $\left(\underset{x \in G_{0}}{\cup} \underline{H_{x}}(A)\right) \circ\left(\cup_{x \in G_{0}} \underline{N_{x}}(A)\right) \subseteq \underset{x \in G_{0}}{\cup} \underline{(H \circ N)_{x}}(A)$, which completes the proof. 


\section{Conclusion}

In this article, we introduced some features of a rough subgroupoid of a groupoid. Let $G$ be a groupoid, $N$ be a normal subgroupoid of $G$. Let $\varnothing \neq A \subseteq G$. The sets $\underset{x \in G_{0}}{\cup} \frac{N_{x}}{}(A), \underset{x \in G_{0}}{\cup} \overline{N_{x}}(A)$ are called lower and upper approximations of $A$ respectively. $N(A)=\left(\cup_{x \in G_{0}} \underline{N_{x}}(A), \cup \underset{x \in G_{0}}{\cup} \overline{N_{x}}(A)\right)$ is called a rough set of $A$ in $G$.A nonempty subset $A$ of a groupoid $G$ is called an $\cup_{x \in G_{0}} \overline{N_{x}}$ rough(normal) subgroupoid of $G$ if the upper approximation $\underset{x \in G_{0}}{\cup} \overline{N_{x}}(A)$ of $A$ is a (normal)subgroupoid of $G$. Similarly a nonempty subset $A$ of $G$ is called an $\underset{x \in G_{0}}{\cup} \frac{N_{x}}{\text { rough }}$ (normal) subgroupoid of $G$ if $\underset{x \in G_{0}}{\cup} N_{x}(A)$ is a (normal)subgroupoid of $G$.

The authors are grateful to the anonymous referees for their careful checking of the details and for their helpful comments that contributed to the improvement of this paper.

\section{References}

[1] X. Liang, D. Li, On Rough Subgroup of a Group, Formalized Mathematics, 17 (2009) 213-217.

[2] Z. Pawlak, Rough sets, Int. J. Comput. Inform. Sci., 11 (1982) 341-356.

[3] N. Kuroki, P.P. Wang, The lower and upper approximations in a fuzzy group, Inform. Sci., 90 (1996) 203-220.

[4] C.Z. Wang, D.G. Chen, A short note on some properties of rough groups, Comput. and Math. with Appl., 59 (2010) 431-436.

[5] R. Biswas, S. Nanda, Rough groups and rough subgroups, Bull. Polish. Acad. Sci. Math., 42 (1994) 251-254.

[6] Z. Wang, L. Shu, The lower and upper approximations in a group, Engineering and Technology, 68 (2012) 8-28.

[7] F. Li, Z. Zhang, The Homomorphisms and Operations of Rough Groups, The Scientific World Journal, Volume 2014, Article ID 507972, 6 pages.

[8] D. Miao, S. Han, D. Li, L. Sun, Rough Group, Rough Subgroup and Their Properties, (2005) 104-113.

[9] G. Oguz, I. Icen, M.H. Gursoy, Lie rough groups, Filomat, 32 (2018) 5735-5741.

[10] N. Bagirmaz, A.F. Ozcan, I. Icen, Topological Rough Groups, Topological Algebra and its Applications, 4 (2016) 31-38.

[11] H. Brandt, ber eine Verallgemeinerung des Gruppenbegriffes, Mathematische Annalen, 96 (1926) 360-366.

[12] A. Grothendieck, T.L. Verdier, Theorie des topos, Lectures Notes Maths, Springer, 1972.

[13] R. Brown, Topology: A geometric account of general topology, homotopy types and the fundamental groupoid, Ellis, Horwood Chichester, 1988.

[14] I.Icen, Sheaves and Local Subgroupoids, arXiv:math/0009086v2.

[15] R. Brown, I. Icen, O. Lie local subgroupoids and their holonomy and monodromy lie groupoids, Topology Appl., 115 (2001) 125-138.

[16] R. Brown, I. Icen, O. Mucuk, Local subgroupoids II:Examples and properties, Topology Apply., 127 (2003) 393-408.

[17] M.R. Buneci, Approximations for uniformly continous functions on groupoids, Surv. Math. Appl., 12 (2017) 219-227.

[18] M.H. Grsoy, H. Aslan, I. Icen, Generalized crossed modules and group-groupoids, Turkish Journal of Mathematics, 41 (2017).

[19] Y.H. Kim, H.S. Kim, J. Neggers, Selective groupoids and frameworks induced by fuzzy subsets, Iran Journal Fuzzy System, 14 (2017) 151-160.

[20] M.R. Buneci, A Urysohn type lemma for groupoids, Theory Appl. Categ., 32 (2017) 970-994.

[21] M.H. Grsoy, I. Icen, Coverings of Structured Lie Groupoids, Hacettepe Journal of Mathematics ans Statistic, 47 (2018) 845-854.

[22] I. Chadja, H. Lnger, Groupoids corresponding to relational systems, Miskolc Math. Notes, 17 (2016) 111-118. 
[23] M.H. Grsoy, I. Icen, The homomorphisms of topological groupoids, Novi Sad J Math., 44 (2014) 129-141.

[24] M.R. Buneci, Morphisms which are continuous on a neighborhood of the base of a groupoid, Studia Sci. Math. Hungar., 42 (2005) 265-276.

[25] P.J. Higgins, Categories and Groupoids, Reprints in Theory and Applications of Categories, 7 (1971) 1-195. 